\title{
EL CRECIMIENTO DE LA FECUNDIDAD FRENTE A LA MODERNIZACIÓN ECONÓMICA Y LAS POLÍTICAS SOCIALES, CHILE 1930-1973
}

\author{
RODRIGO RIVERO \\ Universidad Adolfo Ibañez, Chile \\ rodrigo.rivero@uai.cl
}

\section{RESUMEN}

Este artículo analiza las implicancias del proceso de modernización y la aplicación de políticas sociales sobre los niveles de fecundidad observados en Chile entre 1930 y 1973 . Concretamente, el objetivo es conocer la relación de los factores contextuales -vale decir políticas sociales- y determinantes demográficos, con el crecimiento de la fecundidad que ocurrió, contra todo pronóstico, durante las décadas centrales del siglo XX (1940-1962). Para esto, el artículo calcula indicadores demográficos sobre la base de los datos entregados por los Censos de Población entre 1907 y 2002 y los microdatos censales disponibles en IPUMS-I.

Palabras clave: Fecundidad, Modernización, Políticas sociales, Chile. 


\title{
RISE IN THE FERTILITY RATE AND ECONOMIC MODERNIZATION AND SOCIAL POLICIES IN CHILE, 1930- 1973
}

\begin{abstract}
This paper analyzes the implications of the process of modernization and application of social policies for the fertility rates observed in Chile between 1930 and 1973. Specifically, the objective is to understand the relation of the contextual factors - that is, social policies - and demographic determinants to the rise in the fertility rate which, contrary to all forecasts, occurred in the mid-twentieth century (1940-1962). For this purpose, demographic indicators are calculated using data from the Population Censuses carried out between 1907 and 2002 and the census microdata available in IPUMS-I.
\end{abstract}

Keywords: Fertility, Modernization, Social policies, Chile. 


\section{INTRODUCCIÓN}

El concepto de modernización implica el cambio en el modo de vida del conjunto de una sociedad, que modifica profundamente las complejas relaciones sociales, fundamentalmente, las productivas. Generalmente este cambio en las estructuras productivas implica la transición desde una sociedad de autoconsumo basada en la economía familiar, a la sociedad industrializada de carácter predominantemente urbano, provocando transformaciones culturales importantes entre las que se inscribe el ideal de la familia pequeńa y el cambio del rol de la mujer en la sociedad (Chackiel 2004). Por otro lado, este concepto trae aparejado la idea del desarrollo, tanto en su dimensión económica como social.

En Chile, en la década de 1930, con los gobiernos del Frente Popular (1938-1941) y los radicales (1942-1952), se inicia un ciclo de gobiernos progresistas que llevaron a la práctica los anhelos de modernización. Políticas que tuvieron continuidad a través de los distintos gobiernos de uno u otro sector político hasta la ruptura democrática en 1973. En este sentido, existía una idea ampliamente compartida en la mentalidad de la época: que el desarrollo del país pasaba por la condición de transformar su estructura social y productiva. Un elemento central para alcanzar ese tan anhelado desarrollo, consistía en lograr niveles de bienestar satisfactorios a través de una nueva estructura económica de base industrial, y en segundo lugar, que el Estado se hiciera cargo del bienestar en su dimensión social, en donde las políticas de protección social, como el seguro obrero, o las políticas sanitarias y educativas, jugaron un papel esencial en la mejora de la calidad de vida (Arellano 1985, Ffrench-Davis et al. 2003).

De esta manera, se esperaba una transformación social en línea con el cambio estructural que produce la industrialización y, por otro lado, el cambio demográfico derivado de la urbanización y la extensión de los servicios sociales, en el área de salud y la educación a un creciente número de habitantes (Sunkel 1969). Sin embargo, desde finales de la década de 1940, hasta principios de la década de 1960, justo en los años más intensos del proceso de modernización, la fecundidad aumentó (Rivero-Cantillano 2016) ${ }^{1}$. Las mismas generaciones que fueron protagonistas de las grandes transformaciones que buscaban desarrollar económica y socialmente al país, también fueron protagonistas del crecimiento observado en la fecundidad

1 Entre los años 1946 y 1962 la TBN pasó del 31,7 al 37,7 por mil habitantes respectivamente, nivel equiparable a los observados a principios del siglo XX. Por su parte, la tasa global de fecundidad, entre 1950 y 1960 aumenta de 4,9 a 5,5 hijos por mujer en edad fértil (Rivero-Cantillano 2016). 
durante el siglo XX.

Las interpretaciones de los niveles y cambios de comportamiento en las tendencias observadas históricamente en la fecundidad, involucran varias dimensiones, entre las que se incluyen aspectos biológicos, demográficos, económicos, políticos, sociales, psicológicos y culturales. A estas dimensiones Van de Kaa (1997) ha llamado subnarraciones, que, enfocadas desde distintas orientaciones y perspectivas disciplinarias, habitualmente, adquieren la forma de una teoría verbal. No obstante, a pesar de que nos permiten acumular mayores conocimientos, difícilmente se podrían consolidar en una sola narrativa, para todos los entornos y todas las épocas.

Los trabajos que abordan el cambio en el patrón reproductivo en Chile a lo largo del siglo XX, destacan el papel jugado por el proceso de modernización, las políticas de planificación familiar, el aumento del nivel educativo, así como los cambios sociales en el declive de los niveles de fecundidad a partir de la década de 1960 (Martínez-Pizarro 1998, Rodríguez 2003, Donoso y Carvajal 2009). Sin embargo, no se ha dado la misma importancia a los efectos que estos factores tuvieron sobre el aumento de la fecundidad antes a 1960, aun cuando es sabido que la modernización, a menudo, crea las condiciones que tienden a incrementar la fecundidad (Nag, et. al. 1980).

Para explicar satisfactoriamente este cambio en el comportamiento de la fecundidad durante el proceso de modernización en Chile, seguimos la línea utilizada por Bongaarts (1978), quien analiza el declive de la fecundidad -conocido como Transición de Fecundidad-, mediante los determinantes próximos o intermedios de la fecundidad. De esta manera, nuestra explicación al crecimiento de la fecundidad en Chile, consideraremos dos niveles de análisis, primero, los factores contextuales, aquellos aspectos de la realidad social, política, económica y cultural que vivieron las cohortes responsables del auge, y segundo, los mecanismos a través de los que operan estos factores contextuales, y que determinaron la mayor descendencia de estas cohortes. Estos mecanismos corresponden a los determinantes demográficos del auge de la fecundidad.

Tras la introducción, en la segunda parte de este artículo analizamos el contexto social, político y económico que vivía Chile entre los años 1930 y 1973. En la tercera parte, procedemos al análisis de los determinantes demográficos de la fecundidad, que, influenciados por el contexto, impactaron en el aumento observado en la fecundidad. Por último, ofrecemos las conclusiones. 


\section{LOS FACTORES CONTEXTUALES DEL AUGE DE LA FECUNDIDAD: LAS POLÍTICAS SOCIALES ENTRE 1930 Y 1973}

Un punto central del proceso de modernización fueron las políticas sociales. En este sentido, la dimensión social del nuevo modelo de desarrollo, se concentró en dotar al Estado de un cuerpo legal para el reconocimiento de los derechos y deberes, de empresarios y trabajadores y, por otro lado, crear una institucionalidad que se hiciera cargo del bienestar, donde las políticas de protección social jugaron un papel esencial en la mejora de la calidad de vida.

El avance en las leyes sociales no fue una respuesta unilateral del gobierno o empresarios, sino un largo proceso de negociación no exento de conflicto. Durante décadas de reivindicaciones sociales, del movimiento obrero organizado, y posteriormente organizaciones de campesinos, consiguieron conquistas políticas y legales que les llevaron a lograr un creciente acceso al uso de recursos públicos y al reconocimiento de su ciudadanía como actores sociales con derechos y deberes legalmente constituidos (Olavarría y Parrini 2000). Su legitimidad se fundamenta en la propia lógica del desarrollo, pues corresponden a la dimensión más inclusiva de la modernización al incorporar a amplias masas de población al objetivo nacional de desarrollo (Illanes 2004).

Arellano (1985) divide en dos etapas la formación del sistema de protección social con el hito de 1952 como punto de separación. La primera, se inicia con la aprobación de las leyes sociales en 1924, caracterizándose por la creación de instituciones, y la segunda etapa, a partir de 1952 con la creación del Servicio de Seguro Social (SSS, antigua Caja del Seguro Obrero) y el Servicio Nacional de Salud (SNS), caracterizada por ser una fase de expansión, en la cual el sistema se vio sometido a profundas reformas.

De esta manera, con las leyes sociales se legisló la jornada laboral de ocho horas, supresión del trabajo infantil, reglamentación del contrato colectivo, la Ley de Accidentes del Trabajo y la Caja del Seguro Obrero (CSO), legalización de los sindicatos, la Ley de Cooperativas y la creación de los tribunales de conciliación y arbitraje laboral, se creó el Ministerio de Higiene, Asistencia y Previsión Social y la Cajas de Previsión de Empleados Particulares (EMPART) y la de Empleados Públicos (CANAEMPU). Medidas que se convirtieron en la piedra angular en el proceso de desarrollo y transformación social durante la industrialización. A estas leyes, le siguieron posteriormente otras que configuraron una estructura legal preocupada de la protección y el bienestar de los trabajadores y sus 
familias, como por ejemplo la Ley de Sindicalización Obligatoria de 1928, la promulgación del Código del Trabajo en 1931, la creación de la Caja de la Habitación Popular en 1936, la Ley de Medicina Preventiva de 1937, Ley Madre y Niño en 1937, la creación del Ministerio de Salubridad, Asistencia y Previsión Social en 1939, el establecimiento del Fondo de la Construcción de la Habitación Popular en 1941, el reconocimiento del derecho a sindicalización de los obreros agrícolas en 1967. Sin duda entre las más importantes y que dieron comienzo a una nueva fase, la creación del SSS y SNS en 1952. Este conjunto de leyes abrió una nueva etapa en la relación del Estado con la ciudadanía basada en la configuración de un Estado de bienestar o Estado asistencial (ver Illanes 2004).

El balance hecho del sistema de protección social es positivo, los progresos en desarrollo social fueron notables. Un resultado claro de las políticas sociales de Chile es el mejoramiento significativo del conjunto de indicadores de bienestar social en medio de un contexto de creciente urbanización. La tasa de mortalidad general disminuyó del 20\% en 1940 a $8,7 \%$ en 1970 , por su parte la tasa de mortalidad infantil disminuyó desde los 217 óbitos por mil nacidos vivos a 82 óbitos por mil en el mismo periodo, sin duda un gran éxito (ver Anuario Estadístico, 1940 y Anuario de Demografía, 1970).

\section{LAS POLÍTICAS SOCIALES EN SALUD}

El desarrollo del sistema de salud fue un proceso de integración paulatino de subsistemas de coberturas. En la década de 1920 se crearon una serie de instituciones que cimentaron la constitución del sistema de previsión social en el país, su principal característica fue la fragmentación del sistema. Las políticas aplicadas desde la década de 1920, significaron un primer logro ante las pésimas condiciones de salud de la población chilena. Sin embargo, en la década de 1930 gran parte de la población seguía padeciendo de las llamadas enfermedades sociales. En parte agravadas por los efectos perdurables de la Gran Depresión, la carestía de los artículos básicos de subsistencia y en general por la brecha producida entre los jornales y el costo de vida, incidían en el mal diagnóstico de la salud de la población chilena, los cuadros más comunes comprendían tuberculosis, gonorrea y sífilis, enfermedades que diezmaban a la clase trabajadora y sus familias (Illanes 2004).

En este contexto se aprobó en 1937 la Ley de Medicina Preventiva, que como proyecto reconocía las victorias parciales de los intentos de disminución de la mortalidad y la morbilidad en general que seguían aquejando a grandes sectores de la población. La medicina preventiva se 
basaba en un tratamiento de la enfermedad incipiente para garantizar el retorno del trabajador a su puesto de trabajo.

Durante el Frente Popular la discusión en torno a las políticas de salud, y la reorganización de los servicios, se enlazaban con las necesidades planteadas por el modelo de desarrollo (Molina 2006). A juicio de Allende,

El capital humano, base fundamental de la prosperidad económica, ha sido abandonado a su propia suerte, impidiendo que la población sea compacta, sólida y sana, capaz de producir y de hacer florecer el desarrollo industrial y económico. Los gobiernos anteriores al Frente Popular estimaban postergables los gastos en salud pública sin pensar jamás que la preservación del capital humano constituye la más alta responsabilidad del Estado moderno (1939: 208).

En 1952, tras once años de retraso se aprobó la creación del SNS . En efecto, el proyecto de reforma del régimen previsional y sanitario de los años veinte, fue presentado en 1941 en el congreso por el Ministro de Salubridad del Frente Popular Salvador Allende y estuvo sin tratamiento durante casi una década (Molina 2006). El nuevo aparato de salud se encargó de la centralización y coordinación de la atención médica a los asegurados a lo largo del territorio nacional, "Como resultado, se ampliaba la cobertura social previsional de la Caja del Seguro de 1 millón de beneficiarios a 3 millones" (Illanes 2004: 24).

\section{LAS POLÍTICAS DE PLANIFICACIÓN FAMILIAR Y LA PREOCUPACIÓN MATERNO-INFANTIL}

Hacia la década de 1930 los esfuerzos políticos y médicos se enfocaban, en primera instancia, en atacar los elevados índices de mortalidad y morbilidad que presentaba la población chilena. No obstante, para cumplir con esta prioridad, se hacía cada vez más necesario focalizar las políticas sociales en dos grupos postergados: los niños y sus madres. Si bien, en aquellos años, las malas condiciones de salud eran una característica generalizada de la población chilena, estas adquieren mayor profundidad en estos dos grupos, sobre todo en sectores populares, a través de prácticas poco seguras como herramientas de control de natalidad, como el aborto y el infanticidio, además del abandono y vagabundaje infantil (Rojas 2007). En este sentido, mejorar las condiciones de salud materna e infantil, sirvieron como fundamento a las reivindicaciones de derechos civiles y políticos de las mujeres y, por otro lado, fueron vistas como importantes desafíos para el desarrollo social y económico del país. 
A principios del siglo XX, la asistencia hospitalaria del parto representaba un porcentaje exiguo del número de nacimientos en el país. Solo un poco más de 1.000 mujeres fueron asistidas en la Casa de Maternidad de Santiago en 1899 (Zárate 2007). En el marco de la Ley de Medicina Preventiva y la Ley Madre y Niño, aprobadas en 1937 por el doctor Cruz-Coke en su papel de ministro-, se ponían en marcha beneficios de asistencia maternal, eso sí, contemplaban solamente la cobertura para las mujeres casadas con trabajadores asegurados. Como señala Zárate (2007), en 1937 del total de nacimientos ocurridos en el país, menos de un tercio, el 29,4\% nació en maternidades, el 31,3\% lo hizo en sus domicilios en buenas condiciones, mientras que los entendidos por servicio domiciliario del CSO solo alcanzó al 11,1\% de los nacidos vivos en ese año. El restante $28,1 \%$ nació sin ninguna clase de atención técnica y comodidades. $\mathrm{Si}$ sumamos los porcentajes respectivos, el resultado da que los partos atendidos en condiciones médicas formales en maternidades y por el servicio domiciliario de la CSO alcanzan el 40,5\%.

A pesar de los avances, no fue hasta la creación del SNS, en 1952, que se generó el impulso para la promoción y protección de la salud maternoinfantil, incorporando la atención gratuita para la esposa e hijos menores de 15 años del trabajador asegurado, brindándoles atención prenatal, parto asistido, nutrición y vacunación. La llegada de esta década trajo consigo la preocupación por la salud de las mujeres embarazadas que se mantenía de alto riesgo dadas la frecuencia de los abortos ilegales (Szot-Meza 2002). El avance de la asistencia materna fue notable, como señalan Casas y Herrera (2013), en 1952

[...] solo el $52 \%$ de los partos eran atendidos por profesionales de la salud llegando esta tasa al $40 \%$ o menos en algunas partes del país. Como resultado se produjo un ascenso en la tasa de partos con asistencia profesional hasta el $74 \%$ en 1965 y $81 \%$ en 1970 . También en 1952 se lanzó un programa para brindar suplementos alimenticios a madres lactantes durante las primeras siete semanas después del parto (Casas y Herrera 2013: 62).

Con la entrada en la década de 1960 el SNS entró en una nueva faceta, la planificación familiar. En línea con las nuevas teorías del desarrollo que ligaban la elevada fecundidad con los niveles de pobreza, los países en desarrollo a través del control de la fecundidad buscaron a través de esta vía poner freno a la pobreza e invertir en una mayor paz social (Rojas 1994, Alba 2014). 
De esta manera en 1962 se constituyó el Comité Chileno de Protección de la Familia con el objetivo de asesorar a la dirección del SNS en materia de planificación familiar. Tras un año de funcionamiento, en 1963, se constituyó como una entidad privada bajo el nombre de Asociación Chilena de Protección de la Familia (en adelante APROFA), manteniendo el auspicio y cooperación del SNS (Rojas 1994, Casas y Herrera 2013). Los esfuerzos por dotar al SNS de una política de regulación de la natalidad se concretaron en 1965, diseñada por el departamento de Fomento de la Salud y aprobada por el Consejo técnico del SNS. Al año siguiente la planificación familiar fue incorporada dentro del programa de atención materna, de esta manera las mujeres añaden a la asistencia curativa y preventiva atención en especialidades de ginecología y obstetricia. La implementación de estas políticas se fundamentaba en la necesidad de reducir el número de abortos, el alto índice de partos múltiples, y aumentar el espaciamiento de los nacimientos (Solsona 1986, Casas y Herrera 2013).

En el marco del gobierno de Eduardo Frei Montalva (1964-1970), la planificación se planteó en términos de disminuir el número de niños pobres y abandonados, no siendo explicito el deseo de liberar a la mujer de la carga de criar a un gran número de niños y contribuir a través de esa vía a su desarrollo individual. Sin embargo, la posibilidad de la mujer de disponer de su cuerpo y capacidad reproductiva de forma consciente abrió la puerta a esta nueva realidad, que se materializó gracias a la difusión creciente de las modernas tecnologías anticonceptivas, a través de la política de planificación familiar (Rojas 1994).

Los efectos de esta estrategia de planificación se pueden observar el declive en la Tasa Bruta de Natalidad (TBN) desde mediados de los de los años sesenta (ver Anuario de Demografía 1960-1990). En este sentido, a medida que la planificación familiar abarca mayores contingentes de la población de mujeres fértiles, y esta es adoptada culturalmente, el país moderniza su comportamiento reproductivo, consolidándose las familias pequeñas frente al predominio de las familias numerosas como ocurría en el pasado.

\section{LAS POLÍTICAS SOCIALES EN EDUCACIÓN}

La preocupación por la educación en Chile tiene una larga data. En el proceso de configuración del estado chileno a lo largo del siglo XIX la educación fue un tema central. La temprana promulgación de la Ley General de Instrucción Primaria en 1860, estableció la obligación del Estado de dar educación escolar gratuita en su etapa inicial. Aunque este primer paso significó un avance, fue durante el siglo XX que la educación 
primaria enfocada a las clases populares se consolidó como una política estatal, que sin duda tuvo un gran impacto en los niveles de desarrollo ulteriores. En 1920 la Ley de Instrucción Primaria Obligatoria, sentó las bases del sistema educativo chileno. Luego con la llegada de los gobiernos progresistas, y los planes industrialistas, la educación se tornó en un objetivo primordial, pues hacía falta dotar a la industria del capital humano necesario para su correcto funcionamiento. El Frente Popular una vez en el gobierno abordó el problema con gran preocupación. Es así como Pedro Aguirre Cerda transformó ese desafío en su lema de campaña, Gobernar es Educar. En este sentido, además de su dimensión cívica, de la mano de las políticas industrializadoras cobra gran importancia la dimensión económica de la educación, en tanto que mejorar los niveles de la educación de la población chilena cumplía con los objetivos de transformación de los recursos humanos necesarios para el desarrollo económico en el marco de este nuevo paradigma.

Nuevamente, a pesar de los avances, respecto del reconocimiento por parte del Estado sobre el valor de la educación, la extensión de la cobertura en educación era una tarea aún pendiente hacia mediados del siglo XX. Treinta y dos ańos después de la aprobación de la Ley de Educación Primaria Obligatoria, el Censo de 1952 mostraba una proporción de analfabetismo que daba cuenta de la mala cobertura del sistema educacional, donde el 19,7\% de la población de 15 y más años figuraba como analfabeta. Más preocupante aún es el nivel de alfabetismo de la población en edades formativas. En el censo de 1952, la población entre los 7 y 14 años muestra una proporción de analfabetismo que alcanza el 31\% para el conjunto del país. Claramente el crecimiento de la población en edad escolar se enfrentó ante la escaza cobertura del sistema educativo, que se hizo más evidente en el ámbito rural donde la población entre los 7 y los 14 años de edad muestra un $47 \%$ de analfabetismo en el censo de 1952, prácticamente la mitad de los niños en estas edades.

La incapacidad para llevar a la práctica la legislación aprobada, se evidenciaba en el enfrentamiento entre demanda por educación con la capacidad de los recintos educativos, los que no daban abasto para acoger la población escolar. Una expresión del aumento demográfico, pero también la crecientemente demandaba escolarización por parte de la clase trabajadora para sus hijos fruto de la nueva valoración de la educación como vía de movilidad social (Illanes 2004).

De esta manera, durante la segunda mitad del siglo XX hubo un esfuerzo notable para expandir la cobertura de la educación pública, orientado en dos áreas, los programas de alfabetización de adultos y el aumento de la 
cobertura de la educación entre los niños en edad escolar. El principal instrumento para este objetivo fue la promulgación en 1954 de la ley por medio de la cual se creó el Fondo para la construcción y dotación de establecimientos de la educación pública. Sobre esa base, en la década de 1960 se llevó a cabo un ambicioso plan de expansión de la educación pública. Los resultados de este esfuerzo destacan en el aumento de la cobertura e importancia de la educación pública, el aumento de la tasa de participación escolar, la reducción de los niveles de analfabetismo y una fuerte inversión en la educación técnica, de esta manera se desarrollaron programas educativos adecuados al modelo de desarrollo (Illanes 2004).

\section{MuJER, TRABAJO, MATERNIDAD Y LA FAMILIA EN EL CONTEXTO DE INDUSTRIALIZACIÓN}

En chile las tasas históricas de participación económica de las mujeres, dan cuenta de una baja incorporación al mercado de trabajo durante todo el siglo XX. Respecto al periodo de la industrialización, los datos muestran que la participación económica femenina alcanzó su nivel de participación máximo en el censo de 1952 con un $25,9 \%$, y a partir de ese momento disminuye alcanzando su menor nivel en el censo de 1970 con un 19,7\% de participación (Pardo 1988). En perspectiva histórica a principios del siglo XX la participación de mujeres en el mercado de trabajo era mayor que en la década de 1970 (ver Tabla 1).

Tabla 1:Tasa de participación en la fuerza de trabajo, 12 años y más

\begin{tabular}{cccc} 
Censo & Hombres & Mujeres & Total \\
\hline 1907 & 79,4 & 28,9 & 52,8 \\
1920 & 78,2 & 27,3 & 52,4 \\
1930 & 79,9 & 19,2 & 49,1 \\
1940 & 79,9 & 25,6 & 52,3 \\
1952 & 82,4 & 25,9 & 53,5 \\
1960 & 77,5 & 20,9 & 48,3 \\
1970 & 71,7 & 19,7 & 44,7 \\
1982 & 67,3 & 22,3 & 44,1
\end{tabular}

Fuente: Elaboración propia a partir de Pardo (1988). 
Esta participación femenina se explica por la baja educación que han mostrado las mujeres durante gran parte del siglo XX. El nivel educativo es una de las variables más cercanamente relacionadas con la fecundidad y a la vez con la participación de las mujeres en el mercado de trabajo. Un mayor nivel educativo provee niveles de capital humano más avanzados, lo que a priori, permite acceder a una mayor remuneración en el mercado de trabajo y torna más atractiva la decisión de trabajar fuera del hogar (Larrañaga 2006a, 2006b)

Por ejemplo, observamos para el caso del Gran Santiago las elevadas proporciones de mujeres sin estudios que persisten hasta la última década del siglo XX. Aún en 1980, más de un tercio de las mujeres entre los 25 y 60 años, no tenía estudios. En 1957, esa cifra alcanzaba el 45\% y solo el $2,2 \%$ contaba con estudios superiores (ver Tabla 2).

Tabla 2: Proporción de mujeres de 25 a 60 por nivel de estudios, Gran Santiago

\begin{tabular}{ccccccc} 
Estudios & 1957 & 1960 & 1970 & 1980 & 1990 & 2000 \\
\hline Sin estudios & 44,9 & 44,0 & 38,8 & 35,0 & 26,1 & 17,1 \\
Básica & 40,5 & 40,6 & 37,2 & 34,6 & 24,6 & 16,5 \\
Media* & 12,4 & 13,4 & 19,9 & 26,4 & 42,0 & 42,3 \\
Superior** & 2,2 & 2,0 & 4,1 & 4,0 & 7,3 & 24,1 \\
Total & 100 & 100 & 100 & 100 & 100 & 100
\end{tabular}

* Media: Incluye a recintos educativos cientificos humanistas y técnicos profesionales **Superior: Técnicos nivel superior y con estudios universitarios Fuente: Elaboración propia a partir de Encuesta de Ocupación y Desocupación del Gran Santiago, Centro de Microdatos de la Universidad de Chile.

Estas cifras mostradas por la capital del país nos permiten inferir responsablemente que, en el pasado, la capacitación del capital humano femenino en fue aún peor, así como también nos lleva a ser escépticos con la posibilidad de que la situación del en el resto del país pudiera ser mejor. Por otro lado, podemos observar el fuerte impulso de la educación media a partir de la década de 1980. 
También la podemos relacionar la participación económica de las mujeres con los incentivos legales que ofrece el mercado de trabajo para su participación. En perspectiva histórica, observamos en el inicio de la historia remunerada del trabajo femenino es paralela a la etapa inicial de la producción industrial en Chile, a fines del siglo XIX y principios del siglo XX (Pardo 1988). Los estudios sobre esta temática dan cuenta cómo en un primer momento, desde el Estado a través de la Sociedad de Fomento Fabril (SOFOFA), se señaló la necesidad de aprovechar la mano de obra femenina dedicada fundamentalmente a la realización de oficios poco productivos como la costura, el tejido y el servicio doméstico ${ }^{2}$. Esta mano de obra desaprovechada debería ser formada en las técnicas modernas de producción para de esa forma contribuir al desarrollo nacional desde talleres, fábricas y sus domicilios (Godoy, Díaz y Cardarelli 2009).

De esta manera, la presencia femenina en las industrias abrió el debate acerca de la relación entre el trabajo y la maternidad, la cual fue entendida como un problema. Aunque las medidas para proteger la maternidad de las mujeres obreras fueron resistidas inicialmente, aludiendo a los costes que implicaba para el empleador, se generó un amplio consenso acerca de la conveniencia de su protección, no obstante, la legislación tuvo más en cuenta la condición de madres que la de trabajadora (Godoy, Díaz y Cardarelli 2009). Se consideraba que la presencia de las mujeres en las fábricas como una amenaza al modelo de feminidad-maternidad que caracterizó el discurso dirigido a las familias populares (Godoy, Díaz y Cardarelli 2009, Anseoleaga y Godoy 2013). Esta visión problemática del trabajo femenino se basaba, por un lado, en la degradación de las relaciones patriarcales dentro de la clase obrera y, por otro lado, se argumentaba que aumentaba la presión a la baja de los salarios de los obreros (Godoy, Díaz y Cardarelli 2009).

Con la llegada del Frente Popular, dentro del contexto de la industrialización, se favoreció el modelo de familia nuclear en donde el hombre se asoció a la figura del jefe de hogar, mientras que las mujeres fueron relegadas a un plano subalterno, eminentemente doméstico y dedicado exclusivamente labores reproductivas, modelo conocido llamado como familiar industrial (Valdés y Valdés 2005). En este sentido, un objetivo

2 Hay estudios que muestran una importante presencia femenina en ocupaciones industriales durante las últimas décadas del siglo XIX y primeras el siglo XX, concretamente en el sector textil y confecciones, donde las mujeres correspondían al $80 \%$ de los ocupados (Godoy, Díaz y Cardarelli 2009). Además también se ha documentado una proporción importante de mujeres emprendedoras en el sector textil (Escobar 2015). 
implícito en las leyes sociales fue contribuir a través de su implementación al fortalecimiento del modelo de familia funcional a las necesidades del modelo industrializador, modelo que fue sustentado desde el Estado, pero también desde el movimiento obrero (Olavarría y Parrini 2000). Dichas políticas contribuyeron a fortalecer en los sectores medios de la sociedad chilena y a construir en los sectores populares urbanos y luego rurales este modelo de familia tradicional. La abundancia de gańanes y el flagelo del alcoholismo que afectaba sobre todo a las grandes ciudades a fines del siglo XIX y principios del siglo XX, y el desorden en los nacimientos, corresponden a realidades que fueron vistas como problemáticas pues se asociaba a problemas sanitarios o delincuenciales y como una amenaza para el capital humano (Olavarría y Parrini 2000, Fernández 2006, Fernández et al. 2008). Frente a esta realidad, la industrialización requería de una población trabajadora que perseverara en el trabajo y tuviese necesidad de conservarlo. Estas condiciones se cumplían con los hombres comprometidos con una familia que dependiera de ellos directamente y demandara sus cuidados y protección (Olvarría y Parrini 2000, Valdés y Valdés 2007, Valdés 2005).

La justificación para el fomento de este tipo de familia respondía a las necesidades del proyecto desarrollista, pues se requería incorporar las clases bajas al desarrollo nacional, incorporándolos como capital humano y brindándoles bienestar, no obstante, como señalaba Salvador Allende el capital humano nacional se encontraba "seriamente afectado por el abandono y la imprevisión social” (1939: 208). De esta forma, la constitución adecuada de la familia contribuiría con reducir la ilegitimidad de los nacimientos, fue la vía para remediar a la mala situación médica, demográfica, política y económica que aquejaba a las clases populares (Rosemblatt 1995a).

Por otro lado, coherente con esta visión del trabajo y de la familia, el acuerdo entre los trabajadores, empresarios y el Estado, reconoció la necesidad del salario familiar, el que se materializó que a través del pago de asignaciones familiares que premiaban al hombre casado (discusión que se dio de forma complementaria a la del salario mínimo), el cual debía cubrir las necesidades vitales de consumo familiar. Más allá del alcance real de esta política salarial, está su alcance ideológico, ámbito en la que esta política fue de gran eficacia, desalentando la participación laboral de las mujeres, reforzado el papel de estas como dependientes y dedicadas a labores domésticas (Rosemblatt 1995a, 1995b, Godoy, Díaz y Cardarelli 2009) $)^{3}$.

3 Si bien este fue el discurso hegemónico respecto del trabajo femenino, no fue el 
Así, no es de extrañar que el periodo de declive en la participación laboral femenina durante la industrialización coincida con el periodo de auge de la fecundidad. En este sentido, los datos censales dan cuenta que la tasa de participación económica de las mujeres en las edades más fértiles y más productivas (entre los 20 y 39 ańos) pasó del 32,1\% al 27,1\% entre el censo de 1940 y el censo 1960, recuperándose en el censo de 1982 con un $33,1 \%$ de participación. En cuanto a la tasa participación urbana de este mismo grupo etario, se observa una caída en la participación desde el $37,8 \%$ al 32,8\% entre el censo de 1952 y el censo de 1960 .

\section{FOMENTO DE LA FAMILIA INDUSTRIAL}

Las políticas de vivienda son el reflejo del modelo deseable de familia industrial. Ante la ineficacia de la Ley de Habitación de 1906 y las demandas tanto de los empresarios como de los trabajadores por el déficit de viviendas populares, se creó la Caja de la Habitación Popular en 1936, y cinco años después, el Fondo de la Construcción de la Habitación Popular en 1941(Valdés y Valdés 2005, Valdés 2007).

El diseño de estas viviendas populares se basó fundamentalmente en viviendas unifamiliares, que aseguraba exclusivos para cada uno de los miembros de las familias, y con servicios sanitarios que presentaban al núcleo familiar de epidemias y problemas de salud. Construidas en conjuntos habitacionales en torno a las grandes industrias, ciertas zonas de menor plusvalía, con diseños, superficie y comodidades que fueron variando en el tiempo. En esta época es cuando se comienza a conocer los conjuntos residenciales destinados a empleados y obreros como poblaciones, que contribuyeron a crear una nueva morfología urbana residencial ${ }^{4}$. La casa unifamiliar en los límites de la ciudad, con predios amplios y con todas las condiciones favorables de la higiene moderna, empezó a ser vista como

único. El Movimiento Pro-Emancipación de la Mujer (MEMCH), a lo largo del periodo levantó reivindicaciones en línea con la independencia de la mujer y otras demandas sociales, sin embargo, aunque contribuyó a visibilizar ciertas prácticas discriminatorias, no logró la total independencia del género femenino ni mucho menos equiparar su situación a la de los hombres (Godoy, Díaz y Cardarelli 2009). Aún hoy es posible encontrar con facilidad esta asimetría entre la valoración social del trabajo de hombres y mujeres.

4 A fines de la década de 1960 y comienzos de la de 1970 los diseños de vivienda rural que acompañan la reforma agraria reafirman estos criterios, de la misma manera que los diseños de la vivienda urbana establecían la superficie y distribución de los espacios al interior de ella en función de una familia nuclear (Olavarría y Parrini 2000). 
uno de los objetivos residenciales de los actores medios y medios bajos. Por otro lado, desde las empresas y las instituciones, se legitimó la familia nuclear como el modelo de familia industrial, extendiéndose por amplias capas de la población gracias a los sistemas de protección social estatal (Olavarría y Parrini 2000, Valdés y Valdés, 2005, Valdés 2007).

En este sentido, las políticas sociales de vivienda fueron claramente pro familiares, pues impedían el acceso a la vivienda social a quienes no contaran con la Libreta de Familia otorgada por el Registro Civil tras la realización del matrimonio (Valdés y Valdés 2005, Valdés 2007). De esta manera, se llegó a un mínimo histórico en los nacimientos ilegítimos, entre las décadas de 1930 y 1970, la sociedad chilena mostraba una familia constituida mayoritariamente conforme la ley y una baja histórica de la filiación ilegítima -menos del 16\% - en los años sesenta (Olavarría y Parrini 2000). Por otro lado, este tipo de familia condujo a la consolidación de la separación de esferas masculinas, el trabajo, y las femeninas, la familia y quehaceres del hogar.

Las leyes sociales dictadas en el contexto de la industrialización fueron eficaces en su deseo de modelar a la familia en torno al vínculo matrimonial, frente al "desorden familiar" que reinaba hasta entonces. En esta línea Rosemblatt señala

El fomento al matrimonio a partir de 1938 persuadía a hombres y mujeres de clases populares para que se apartaran de las formas familiares erráticas y disfuncionales asociadas al pasado. [...] de modo que bajo los gobiernos de Frente Popular, las distancias entre las normas familiares y los comportamientos sociales tendieron a acortarse (1995b: 183).

\section{DETERMINANTES DEMOGRÁFICOS DEL AUGE DE LA FECUNDIDAD EN CHILE DURANTE LA MODERNIZACIÓN}

Disponer de datos para conocer cada causa potencial del crecimiento de la fecundidad es un camino con múltiples obstáculos, pues para muchas de estas causas no hay cuantificación para nuestro periodo de estudio. No obstante, podemos examinar variables demográficas de especial importancia que se asocian al nivel de fecundidad y que en el contexto de modernización sufren cambios considerables. Algunas de estas variables que examinaremos están incluidas en el modelo de las variables próximas o variables intermedias, a través de las cuales los factores socioeconómicos y culturales afectan a la fecundidad (Davis y Blake 1956, Bongaarts 1978, Rosero-Bixby 1996, Bay et. al. 2003, Perez-Brignoli 2010). Aun cuando 
el modelo de las variables próximas se ha utilizado para explicar el declive observado a partir de la década de los sesenta, es de vital importancia seguir estas variables en un sentido histórico -cuando sea posible- con el fin de conocer cuál fue su comportamiento en el escenario previo al inicio de la tasa de fecundidad, que como ya hemos mencionado se caracteriza por un crecimiento en los niveles de fecundidad. El supuesto que está detrás de este ejercicio es que, si el comportamiento de estas variables determina el declive de la fecundidad, una dinámica diferente, puede igualmente, estar detrás del crecimiento de la fecundidad.

\section{NUPCIALIDAD, FAMILIA Y FECUNDIDAD}

Nupcialidad y fecundidad, corresponden a dos variables demográficas en las que intervienen las decisiones conscientes de los individuos y parejas. Detrás de la opción de contraer matrimonio se alternan una serie de decisiones respecto a la vida productiva y reproductiva de la pareja que podemos resumir en la creación de una familia, cuyo objetivo principal será la descendencia ${ }^{5}$. Por lo tanto, este proceso decisorio se da en un terreno que involucra la economía familiar, la cual sin duda se ve influenciada por el contexto económico, social y cultural en el que se desarrolla.

Para Malthus el matrimonio constituía el primer determinante de los niveles de fecundidad y la clave de los obstáculos preventivos al crecimiento demográfico excesivo (Zavala de Cosío 1992). Siguiendo esta línea de controles preventivos, Davis (1963) plantea, por un lado, al celibato y el aplazamiento de la edad con la que se contrae el matrimonio, y por otro, el control de la fecundidad, como respuesta efectiva a las presiones demográficas surgidas del descenso de la mortalidad. La teoría de Davis ha sido confirmada por la historia de Europa, donde las poblaciones usaban el aplazamiento del matrimonio para regular su crecimiento (Rosero-Bixby 1992). Sin embargo, en América Latina, la nupcialidad no ha cumplido con esta función. A diferencia de la experiencia europea, la nupcialidad como método de control de la fecundidad o regulación demográfica, nunca ha sido un patrón social y culturalmente aceptado (Zabala de Cosío 1992). De modo que si esta estrategia social propuesta por Davis funcionara en el caso de Chile, observaríamos que como respuesta a las presiones

5 Si bien en la actualidad existen diversas tipologías de familias, las generaciones que abarca este estudio responden al modelo de familia que hoy se conoce como tradicional, compuesta por un padre, generalmente jefe de familia, una madre encargada de las labores domésticas y los hijos. En su versión extendida esta puede abarcar a los abuelos u otros familiares cercanos. Por otro lado, el objetivo primordial de la familia tradicional era la procreación (Quilodrán 2011). 
demográficas derivadas de la rápida reducción de la mortalidad a partir de la década de 1930, se produciría un cambio en el patrón de nupcialidad tendentes a la reducción de los niveles en la tasa bruta de nupcialidad, así como el retraso de la edad a la que se contrae el vínculo matrimonial, sin embargo, observamos todo lo contrario.

En este sentido, Rosero-Bixby (1992) evalúa el impacto de la nupcialidad en la fecundidad, en los cinco países de la región con variaciones significativas en la tasa global de fecundidad durante la década de 1950 (El Salvador, Costa Rica, Panamá, Guatemala y Chile). Su estimación para el caso de Chile, señala que el 93\% del cambio en la tasa global de fecundidad durante la década de los cincuenta es explicado por la existencia de un boom matrimonial, reconociendo de esta manera la relación entre la nupcialidad y la fecundidad. Por otro lado, como hemos visto anteriormente, las políticas de incentivo a la formación de familias bien constituidas o familia industrial, logró hacer disminuir los nacimientos ilegítimos, que alcanzó su nivel más bajo en la década de 1970, lo cual da cuenta que la fecundidad se desarrolló en una proporción creciente dentro del matrimonio (Rosemblatt 1995b).

El Censo de Población de 1952, fue el primer censo en Chile en recoger datos de las uniones consensuales bajo la categoría de convivientes. En este censo, las mujeres entre los 15 y 49 años de edad que declararon su estado civil como convivientes representan un $7,1 \%$ del total de las uniones (casadas más convivientes), a partir del cual se observa un declive en la proporción de convivientes que alcanzó el 5 \% en el censo de 1970, su nivel más bajo en todo el siglo XX.

La trayectoria de la tasa bruta de nupcialidad, muestra una tendencia de crecimiento durante gran parte de la primera mitad del siglo XX (ver Gráfico 1). Como podemos observar, entre las décadas de 1940 y 1960, a pesar de las fluctuaciones, el comportamiento de la nupcialidad se mantuvo en los mayores niveles observados, en torno a los $8 \%$ matrimonios, salvo por el peak de fines de los años veinte y principios de los treinta. Posteriormente en la década de los ańos ochenta se observa el contraste respecto del pasado de la nupcialidad, cuando la emergencia de las uniones consensuales hizo cambiar la tendencia observadas en las décadas previas, decantándose decididamente por el declive durante la década de 1990. 
Gráfico 1: Tasa bruta de nupcialidad, 1900-1999

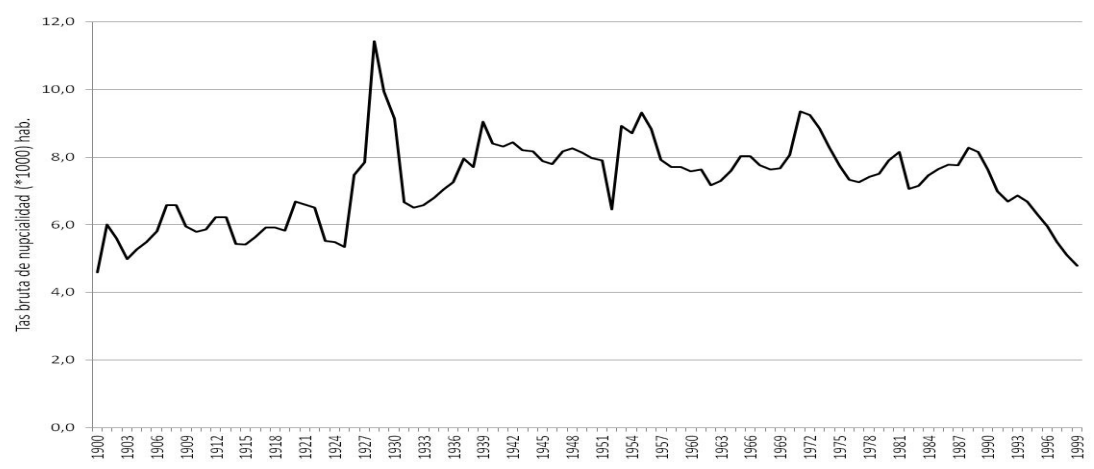

Fuente: Elaboración propia a partir de Anuarios Estadísticos y Censos de población INE.

La proporción de mujeres casadas por grupos de edad, alcanza el valor máximo en el grupo de edades entre 35 y 39 años, para después comenzar a descender lentamente. Este descenso se debe, a divorcios, separaciones y a muertes del cónyuge. De este modo, el complemento de los porcentajes con respecto al $100 \%$ equivale al porcentaje de mujeres solteras, viudas, divorciadas o separadas dentro de ese grupo de edad (ver Gráfico 2).

Gráfico 2: Proporción de mujeres casadas por grupo de edad. Censos 1920-1992

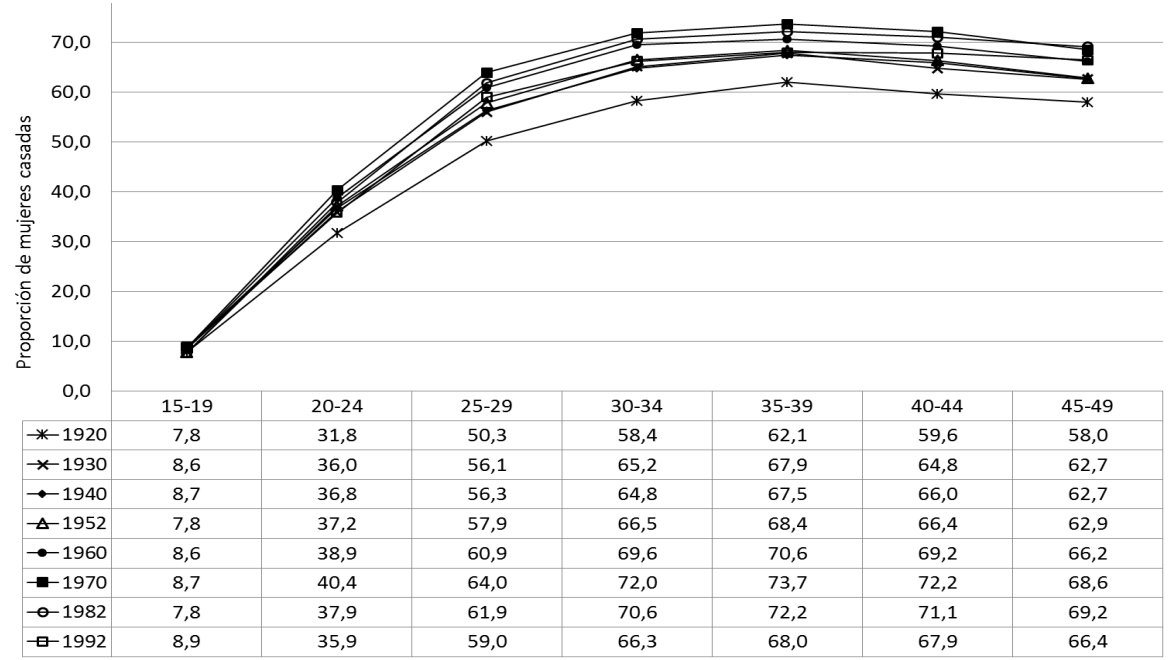

Fuente: Elaboración propia a partir de Censos de Población, INE.

Si interpretamos de forma longitudinal la información de momento que nos entregan los censos, comprobamos que el aumento en la proporción de mujeres casadas en los censos de 1920 a 1970, coincide con el periodo 
reproductivo de aquellas cohortes que vieron aumentar su descendencia final (DF) ${ }^{6}$, las cohortes de 1910 a 1925 , que inician su vida reproductiva en 1925 y la finalizan en 1974 .

En las poblaciones con escaso control de la fecundidad, como ocurre en Chile previo a la década de 1960, el tiempo de exposición al riesgo de embarazo prevalece ampliamente sobre otros efectos que puedan intervenir en la relación de la nupcialidad con la fecundidad, al mismo tiempo que este efecto se vuele irrelevante en sociedades poseedoras de una cultura de control de la fecundidad y planificación de la fecundidad ampliamente difundida (Rosero-Bixby 1992).

El número promedio de años que una mujer vive en matrimonio durante su etapa fértil, una medida similar a la esperanza de vida, se podría catalogar como esperanza de vida en unión de una mujer durante su vida fértil. De acuerdo con las cifras de mujeres casadas disponibles en los censos de 1920 a 1992, podemos observar un aumento considerable de la proporción de años de vida fértil que las mujeres viven en matrimonio entre los censos de 1920 a 1970 (ver Gráfico 3).

Gráfico 3: Tiempo vivido en matrimonio (en porcentajes de años de vida fértil de la mujer). Censos 1920-1992

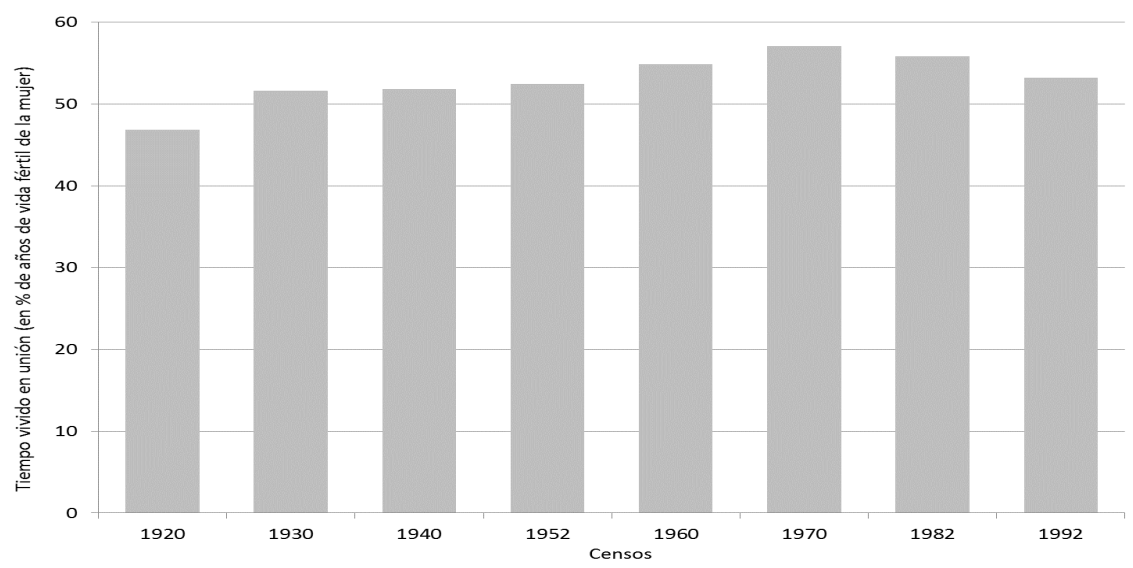

Fuente: Elaboración propia a partir de Censos de Población, INE.

La proporción de tiempo de vida fértil que las mujeres vivieron dentro del matrimonio pasó desde el $47 \%$ al 57\% entre 1920 y 1970 . En este tiempo

6 Descendencia final, también llamada paridez acumulada, es un indicador que se refiere al número promedio de hijos que tendría una cohorte o generación hipotética de mujeres al final de su periodo reproductivo o vida fértil. 
promedio intervienen dos factores, las interrupciones del matrimonio, que como hemos visto no es significativo para antes de los cuarenta ańos y, por otro lado, el adelantamiento de la edad media al matrimonio. La combinación de estos efectos, culmina en la ampliación del tiempo vivido en unión durante la vida fértil de las mujeres que, en un contexto de ausencia de control de la fecundidad, actúa contribuyendo al aumento de la DF de las generaciones que fueron protagonistas de estos cambios.

La edad a la cual se produce la primera unión es de vital importancia para entender las fluctuaciones ocurridas en la fecundidad, mientras antes se produzca la unión mayor será el tiempo de exposición al riesgo del embarazo. A través del Singulate Mean Age at Marriage $(\mathrm{SMAM})^{7}$, y la edad mediana al primer matrimonio o unión (en adelante, EMPM) ${ }^{8}$, podemos comprobar un descenso, o más bien adelantamiento, de la edad en ambos indicadores, entre 1920 y 1970, tanto en hombres como en las mujeres, estabilizándose a partir de los años setenta y retrasando levemente hacia 1992 (ver Tabla 3)9.

7 SMAM expresa el promedio de años de vida en estado de soltería entre aquellos que se casan antes de los cincuenta años (Binstock y Cabella 2011). En este artículo hemos incluido en el cálculo del SMAM a todas aquellas personas casadas y en unión consensual no necesariamente legalizada, por lo que debe entenderse como la edad a la primera unión. Por otro lado, en su elaboración utilizamos los datos que figuran en los Censos de Población realizados por el Instituto Nacional de Estadísticas (INE) entre 1920 y 1992. Respecto al estado civil por sexo y edad la unión consensual distinta al matrimonio comienza a ser registrada en los censos de población a partir de 1952.

8 La edad mediana al primer matrimonio es la edad a partir de la cual el 50\% de los hombres o las mujeres en edad de contraer matrimonio o unión, efectivamente han contraído el vínculo, quedando otro 50\% soltero. Tanto EMPM como SMAM, se calculan sobre la base de la proporción de soltería, por lo que en este artículo a partir del Censo de 1952 consideramos a la población en unión consensual en el mismo estatus que si hubiesen contraído matrimonio legal.

9 Los Censos previos a 1952 no contabilizaban las uniones fuera del matrimonio (convivientes) por lo que puede existir una sobreestimación de la categoría de mujeres solteras. En este sentido, es probable que para los censos de 1930 y 1940 puede haber una sobreestimación aproximadamente del 3\% de las mujeres solteras (esta cifra la obtuvimos asumiendo la tasa de crecimiento geométrico de las conniventes en el periodo intercensal 1950-1960, 1,8\%). 
Tabla 3: EMPM y SMAM censos de 1920 a 1992

\begin{tabular}{ccccc} 
& \multicolumn{2}{c}{ EMPM } & \multicolumn{2}{c}{ SMAM } \\
\cline { 2 - 5 } Censo & Hombres & Mujeres & Hombres & Mujeres \\
\hline 1920 & 28,8 & 23,7 & 28,4 & 25,5 \\
1930 & 26,7 & 23,4 & 28,1 & 24,9 \\
1940 & 26,9 & 23,1 & 28,7 & 24,7 \\
1952 & 25,8 & 22,5 & 27,0 & 23,7 \\
1960 & 25,4 & 22,5 & 26,4 & 23,5 \\
1970 & 25,0 & 22,4 & 25,8 & 23,4 \\
1982 & 25,1 & 22,7 & 25,7 & 23,6 \\
1992 & 27,3 & 22,4 & 25,8 & 23,4
\end{tabular}

Fuente: Elaboración propia a partir de Censos de población (INE 19201992).

Por lo tanto, comprobamos que, hasta la década de 1970, tanto los hombres como mujeres en edades reproductivas, acceden en mayor proporción al matrimonio o unión, además, lo hacen de forma más temprana que generaciones anteriores. Por otro lado, el adelantamiento de la edad con la que se accede a la vida conyugal, muestra un cambio en las preferencias de los individuos, existiendo una mayor disponibilidad a iniciar la convivencia en pareja o a la formación de familias, lo que puede estar motivado por una mejora en las expectativas de las personas al unirse. Mientras que el SMAM para 1920 es de 25,5 años, en 1970 es de 23,4 ańos, lo que refleja un adelantamiento de 2,1 ańos a la edad de la unión. En los censos posteriores a 1970, observamos el escenario opuesto, la tendencia observada en el SMAM como en la EMPM es la postergación a la edad a la cual se contrae por primera vez el vínculo conyugal ${ }^{10}$.

10 Una alternativa para compensar la falta de información sobre uniones extramatrimoniales y que puedan estar sesgando los resultados de SMAM y EMPM en los censos previos a 1952, es observar la proporción de mujeres solteras al cabo de la vida fértil $S(50)$ que corresponde a la proporción promedio de soltería en los grupos de edad 45-49 y 50-54. La soltería es el único estado civil irreversible, por lo tanto, habiendo contabilizado adecuadamente los otros estados civiles, incluyendo 
Ambos escenarios, el adelantamiento, como la postergación del matrimonio o unión, están influenciados por el contexto o acervo cultural en el cual se desarrollan estas dinámicas, además de las concomitantes socioeconómicas. En el primer caso, el adelantamiento, se da en un contexto de importantes mejoras en las condiciones de bienestar y las expectativas de vida. De esta manera, las mejoras introducidas por el proceso modernizador convivieron con la persistencia de costumbres aún conservadoras o tradicionales respecto al papel de la mujer dentro de la familia y de la sociedad. El segundo, la postergación, se da en un contexto en el que nuevos valores sociales afectan al comportamiento reproductivo de las parejas y la formación de las familias. Además, se profundiza el cambio significativo en la naturaleza del vínculo, cobrando mayor protagonismo las uniones libres y la planificación familiar (Binstock y Cabella 2011).

\section{EL RETROCESO DE LA INFECUNDIDAD}

La proporción de la infecundidad, o lo que es lo mismo, la proporción de mujeres que han llegado al final de su vida reproductiva sin haber tenido descendencia alguna, ha experimentado cambios a través de las generaciones. Esta proporción es el complemento a uno de la proporción de mujeres que tuvieron al menos un hijo antes del final de su vida fértil (Nicolau et. al. 2010) ${ }^{11}$.

Los niveles de infecundidad observados van desde el 16\% de las mujeres de la generación de 1910 a un 7\% en la generación de 1955, menos de la mitad que a principios del siglo. En cuanto a las mujeres alguna vez unidas la infecundidad lógicamente es sensiblemente menor, debido al mayor riesgo de embarazo por efecto de la unión. En comparación a países europeos, los niveles de infecundidad observados en las cohortes son bajos (Devolder y Merino 2004, Morgan 1991). La mayor fecundidad y, por ende, la menor infecundidad observada en Chile, se enmarcan dentro de

las uniones consensuales -datos que podemos disponer a partir del Censo de 1952 en adelante- no existen riesgos de sobrestimación de la proporción de soltería. Los resultados del S(50) para los censos de 1952 a 1982, al estar calculados sobre la base de los grupos 45-49 y 50-54, abarcan las cohortes de nacimiento desde 1900 hasta 1935. En el censo de 1952, la proporción de mujeres solteras a los 50 años de edad (S50) alcanzaba el 16\%. En los censos posteriores esta proporción pasó a 14,7\% en el censo de 1960, 12,9\% en el censo 1970 y 12,4\% en el censo de 1982, declive que coincide con el aumento de la DF en las cohortes 1910 y 1925, también coincide con el aumento en la proporción de nupcialidad y respalda de esta manera la tendencia que observamos en los indicadores de SMAM como en EMPM.

11 Ver Rivero-Cantillano (2016), la infecundidad vendría dada por 1-. 
la tendencia y niveles de DF observados en la mayor parte de los países de la región (Reher y Requena 2014).

Gráfico 4: Infecundidad de las cohortes de 1910 a 1960 (todas y alguna vez unidas)

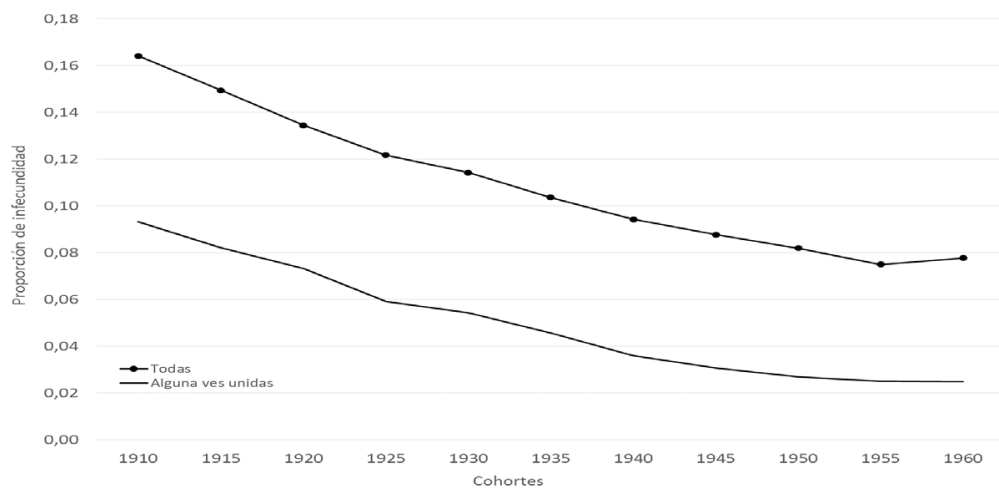

Fuente: Elaboración propia a partir de IPUMS-I.

Las causas detrás de esta reducción pueden asociarse a una suma de factores que influyen de manera positiva en la reducción de la infecundidad, como las mejoras en la salud materna gracias a la erradicación de malas prácticas en el momento del parto o condiciones adversas a las que deben hacer frente las mujeres en su periodo de gestación, lactancia o reproductivo en general (Ortiz et. al. 1978, Zárate y Godoy 2011). Los efectos del control de enfermedades contagiosas o de transmisión sexual como posible causa de la alta infecundidad de las cohortes en las cohortes de principios del siglo XX, justificará el descenso posterior (Zárate 2011).

Por otro lado, los efectos del crecimiento de las uniones en las cohortes de la primera mitad del siglo XX, es coherente con la tendencia mostrada por la infecundidad. Como hemos mencionado anteriormente, en el censo de 1952, la proporción de mujeres solteras a los 50 ańos de edad, es decir aquellas mujeres que acabaron su periodo reproductivo en estado de soltería, alcanzaba el $16 \%$, proporción que se redujo hasta el $12,4 \%$ en el censo de 1982, declive que coincide con el aumento de las uniones, el aumento de la DF y la reducción de la infecundidad en las cohortes de 1910 a 1925.

A través de los niveles educativos, podemos comprobar las diferencias en la infecundidad al interior de la sociedad chilena. Como sucede habitualmente cuando se realiza este tipo de análisis, afloran las desigualdades intrínsecas a la conformación de las sociedades, en este caso asociadas a los niveles de infecundidad y las tendencias generacionales 
observadas. Al igual que los nuevos hábitos o costumbres respecto de los comportamientos reproductivos siempre comienzan por los sectores sociales más aventajados (Martínez-Pizarro 1998), en el comportamiento de la infecundidad podemos observar el grado de difusión de las mejoras introducidas por la modernización a través de las distintas capas sociales (ver Gráfico 5).

Comprobamos que cuanto más bajo es el nivel educativo de las mujeres menor es la infecundidad. Entre las mujeres con estudios primarios incompletos, observamos que la infecundidad en la generación de 1910 se sitúa por debajo del $15 \%$. Por lo general, los sectores sociales menos favorecidos son aquellos que presentan una mayor fecundidad, producto del estilo de vida tradicional asociado al medio rural como espacio predominante en el cual se desarrolla la vida productiva y reproductiva de estas mujeres, pero también, por falta de acceso o desconocimiento de métodos de control de la fecundidad, con lo cual los cambios en este grupo se dan más tarde o de forma más lenta que los sectores sociales más avanzados. En este sentido la mayor caída en la infecundidad entre las generaciones de 1910 y 1925 lo observamos en las mujeres con estudios primarios completos, pasando de un $20 \%$ de infecundidad a un $14 \%$.

Gráfico 5: Diferencias en la infecundidad por nivel educativo, cohortes de mujeres de 1910 a 1960

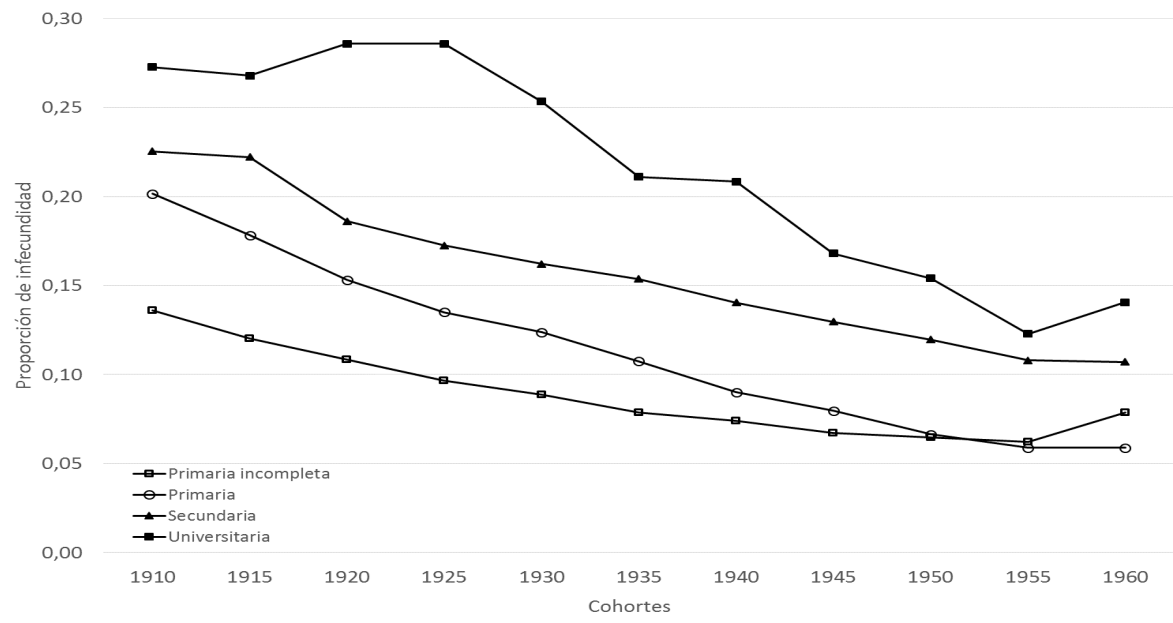

Fuente: Elaboración propia a partir de IPUMS-I.

De la comparación entre sectores socioeconómicos resultan diferencias no solo en los niveles de infecundidad sino también en las tendencias. Observamos que en las mujeres con educación secundaria completa la reducción de la infecundidad no se manifiesta de forma clara hasta la 
generación de 1915, a partir de esta se reduce de forma constante, aunque conservando un nivel más elevado respecto a los otros dos niveles educativos inferiores. Por su parte, las mujeres con estudios universitarios presentan los mayores niveles observados de infecundidad, superiores al $25 \%$ en las generaciones de 1910 y 1915 . Las generaciones de 1920 y 1925 ven crecer el porcentaje de infecundidad, niveles y tendencias que no se observa en los otros niveles educativos.

Por otro lado, en las cohortes de 1910 a 1930, la reducción de la infecundidad fue una dinámica que se dio junto al aumento observado en la DF (ver Gráfico 6). Como hemos visto anteriormente, en estas cohortes, el aumento de la proporción de mujeres casadas y el adelantamiento tanto en la EMM como en el SMAM, se dio junto a la ausencia de prácticas para el control de la fecundidad. A partir de la cohortes de 1930 y subsiguientes observamos un cambio en la dinámica, pues como hemos indicado, las cohortes posteriores a 1930, pero con mayor intensidad a partir de la cohorte 1935 en adelante, reducen paulatinamente su DF a pesar de aumentar las probabilidades de ser madre, puesto que la caída de la fecundidad se debe a la disminución de la probabilidad de tener más de tres hijos (Rivero-Cantillano 2016).

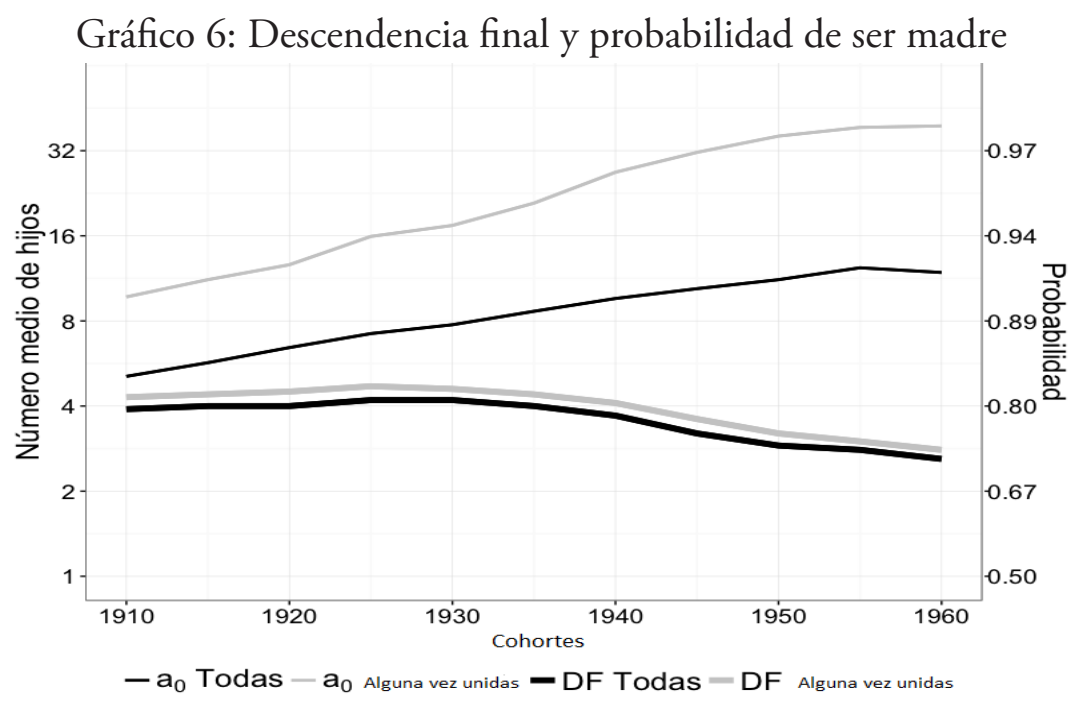

Fuente: Elaboración propia a partir de IPUMS-I.

Las diferencias entre las tendencias seguidas por la infecundidad y la DF, se dio gracias al cambio en el comportamiento reproductivo de las mujeres que se observa de forma incipiente a partir de las cohortes nacidas en de la década de 1930 y se profundiza en las siguientes generaciones. 
Las cohortes de mujeres que nacieron a mediados de la década de 1930, fueron las primeras que se vieron expuestas a los planes de control de la fecundidad, al uso de la píldora anticonceptiva y los programas de planificación familiar. En otras palabras, son las primeras generaciones de mujeres que desean tener menos hijos y controlan su fecundidad y, en consecuencia, el tamaño de las familias a través de métodos modernos y de forma voluntaria. Por esto, la reducción de la infecundidad no se tradujo en una mayor DF.

\section{REDUCCIÓN DE LA MORTALIDAD UNA PRESIÓN AL ALZA DE LA FECUNDIDAD}

El espectacular declive de los niveles de mortalidad a todas las edades actuó aumentando la sobrevivencia de forma transversal. En los primeros años de la transición demográfica, las ganancias en años de vida que conlleva la reducción de la mortalidad se concentran en las edades más jóvenes, específicamente en los primeros cinco años de vida, pues el componente infantil de la mortalidad sin duda fue determinante en la rápida reducción de los niveles agregados de mortalidad (Rivero-Cantillano y Spijker 2015). Como ya hemos mencionado, la reducción de la mortalidad infantil es tal vez uno de los indicadores más elocuentes de los avances ocurridos al interior de la sociedad chilena atribuibles a la modernización. Podemos observar la relación entre mortalidad y fecundidad contrastando la DF de las generaciones con el número de hijos sobrevivientes ${ }^{12}$. En este sentido, la mayor sobrevivencia contribuye al crecimiento de la descendencia final de las mujeres, entre las generaciones de 1910 a 1925 (ver Gráfico 7.).

12 Los datos de IPUMS-I incluyen la pregunta respecto de los hijos sobrevivientes al momento del censo (CHILDSURV), a las mujeres mayores de quince años. Las respuestas pueden estar afectadas por el sesgo de la mortalidad pues las mujeres mayores tendrán una menor sobrevivencia al estar estos expuestos por mayor tiempo a los riesgos de la mortalidad. Sin embargo, estudios en esta línea señalan que las diferencias en este sentido deben ser relativamente pequeńas, porque en las primeras fases de la transición demográfica la mayoría de la mortalidad ocurre en los primeros años de vida. Por esta razón, es un indicador aproximado del impacto de la sobrevivencia de los hijos en el boom de la fecundidad (Reher y Requena 2014). 
Gráfico 7: Descendencia final e hijos sobrevivientes, cohortes 1910-1960

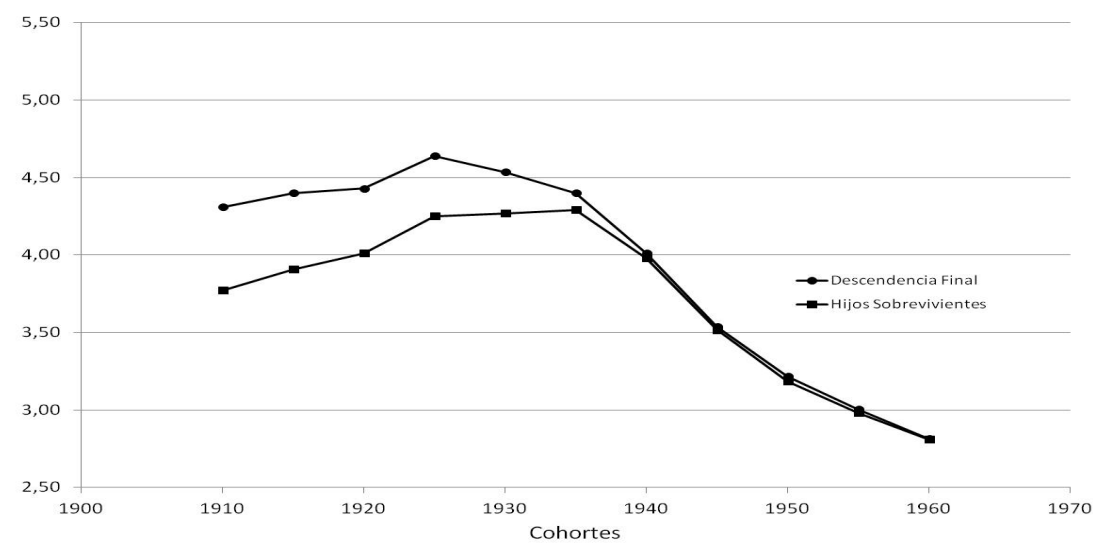

Fuente: Elaboración propia a partir de IPUMS-I.

Los mayores contrastes entre la DF final y los hijos sobrevivientes de las generaciones de mujeres alguna vez unidas podemos observarlo en aquellas generaciones que fueron protagonistas de la modernización y que sus periodos reproductivos coinciden con los años de mayor reducción de la mortalidad infantil. Podemos decir que existe un fuerte vínculo entre la DF y la mortalidad en estas generaciones previas a la transición de la fecundidad y que desarrollan su periodo reproductivo en medio de las grandes transformaciones sanitarias modernizadoras. Por otro lado, a partir del declive de la DF las diferencias entre los hijos nacidos y los hijos sobrevivientes se reducen considerablemente hasta que en la generación de 1940 no se observan discrepancias, certificando el éxito en la lucha contra la mortalidad infantil.

Simultáneamente a la batalla en las condiciones sanitarias que afectan a la mortalidad infantil, la expansión de la educación y la mejora del nivel educativo de las generaciones de mujeres, contribuyen a la reducción de esta brecha, cuanto mayor es el nivel educativo, menor es la diferencia entre los hijos tenidos vivos y los hijos sobrevivientes (Zárate y Godoy 2011). De esta manera, se articula la relación entre el nivel educativo y la sobrevivencia de los hijos.

A través de la discrepancia entre los hijos tenidos y los sobrevivientes por nivel educativo de las madres, podemos observar el diferencial trato que da mortalidad a las distintas capas sociales (ver Gráfico 8). 
Gráfico 8: Descendencia final e hijos sobrevivientes por nivel educativo, cohortes 1910-1960 (alguna vez unidas)
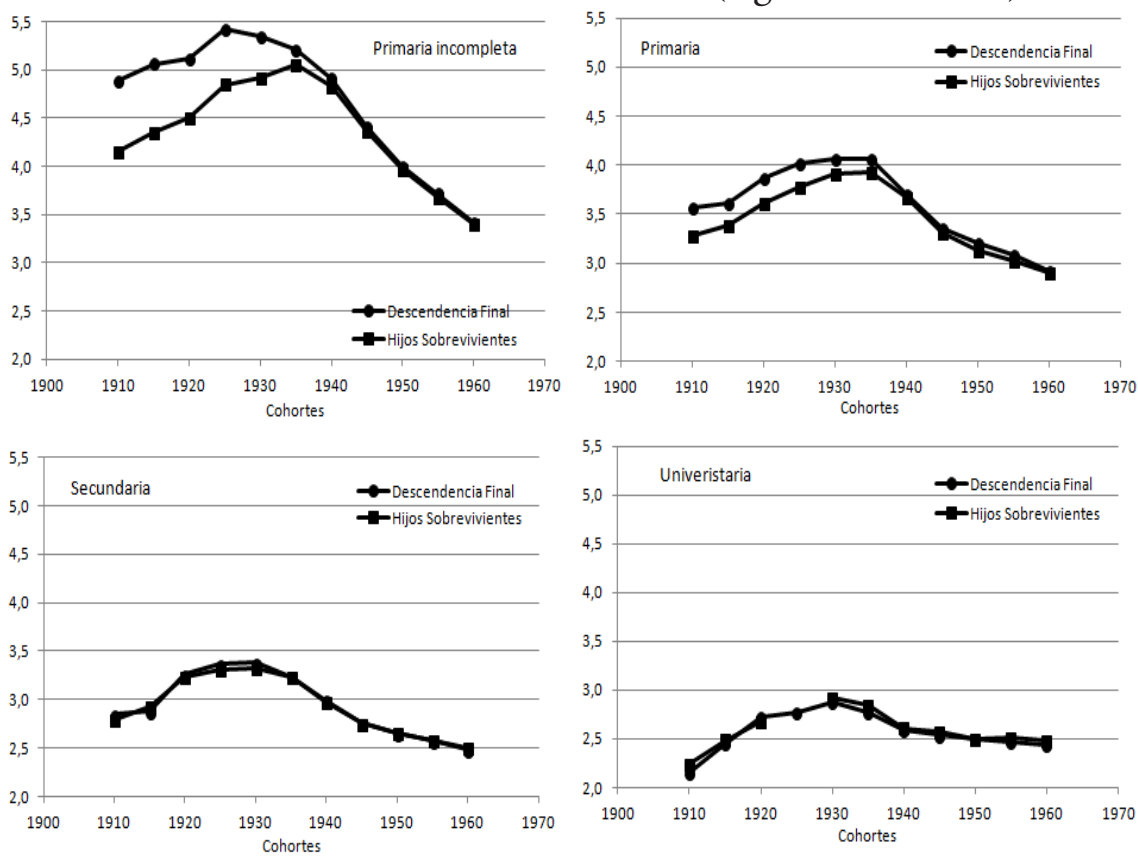

Fuente: Elaboración propia a partir de IPUMS-I.

Como podemos observar, mientras que en el nivel educativo encontramos importantes brechas entre los hijos tenidos vivos y los hijos sobrevivientes entre las generaciones de 1910 a 1925, estas son inexistentes en los niveles educativos más altos. Por otro, lado entre las mujeres con primaria completa e incompleta se observa la reducción de la discrepancia entre las generaciones, igualándose en la generación de 1940.

Sin embargo, el crecimiento de la DF transversal a todos los niveles educativos entre las generaciones de 1910 a 1925, incluso en aquellos niveles donde no se observan discrepancias entre los hijos tenidos y los hijos sobrevivientes en estas generaciones. Es evidente que la mayor sobrevivencia impacta sobre los niveles de fecundidad, y que esta tiene un impacto mayor en las capas sociales más bajas. No obstante, el crecimiento en los niveles de fecundidad en ausencia de diferencia entre los hijos nacidos y los hijos sobrevivientes, nos confirma que no es la única variable que presiona al alza a los niveles de fecundidad en las generaciones previas a la transición de fecundidad. Por otro lado, durante el periodo de discrepancia no se observa una reacción que tienda a disminuir la fecundidad, sino hasta el momento del declive. 


\section{CONCLUSIONES}

En este artículo hemos contribuido a la compresión del crecimiento de la fecundidad durante el periodo de la industrialización, estableciendo el vínculo entre los factores del contexto y los determinantes demográficos que desencadenaron el cambio observado en la fecundidad.

El modelo de desarrollo basado en la industrialización, se asoció a objetivos como la modernización institucional y la transformación social. Mejorar las condiciones de vida era necesario no tan solo desde un punto de vista humanitario, sino también era una tarea fundamental para generar el capital humano requerido por el nuevo modelo de desarrollo. Para ello se puso en marcha una nutrida gama de políticas sociales que buscaban cumplir con este objetivo. Sus resultados saltan a la vista y son fácilmente comprobables en los indicadores de desarrollo económico y social. No obstante, aquí ofrecemos una nueva interpretación de sus efectos sobre la población chilena, el papel jugado por estas políticas modernizadoras en el aumento de la fecundidad.

Respecto de los determinantes demográficos de la fecundidad, observamos los siguientes cambios asociados al aumento de la fecundidad entre los años 1947 y 1962:

- $\quad$ Aumento de la tasa bruta de nupcialidad entre 1920 y 1940 , seguido por la estabilidad en niveles elevados entre 1940 y 1970, periodos que coinciden con la vida fértil de las cohortes responsables del auge de la fecundidad (las cohortes de 1910 a 1925 que vivieron sus periodos reproductivos entre 1925 y 1974). En Chile, al igual que en la mayor parte de América Latina, el vínculo matrimonial no ha sido utilizado como un método de control de la fecundidad, por lo que una mayor nupcialidad se puede asociar a una mayor fecundidad.

- Sumado a lo anterior, entre los censos de 1920 y 1970 detectamos una mayor exposición al riesgo de embarazo a través de los indicadores EMPM y SMAM. Estos indicadores evidencian el adelantamiento en la edad de acceso al primer matrimonio, extendiendo así la proporción de ańos de vida fértil que las mujeres viven en unión. Por otro lado, volvemos a establecer aquí la coincidencia con el periodo reproductivo de las cohortes responsables del auge de la fecundidad (1910-1925).

- Se observa la reducción de la infecundidad con gran intensidad entre las cohortes de 1910 y 1925, producto de la mayor exposición al riesgo de embarazo y de las mejoras en la atención materno-infantil 
en la década de 1950, resultando así una mayor probabilidad de ser madre. Por otro lado, esta reducción de la infecundidad fue acompañada por el aumento de la DF hasta la cohorte de 1930.

- Respecto de las diferencias en los niveles de infecundidad entre las capas sociales, observamos que los niveles educativos más bajos muestran una menor infecundidad producto de su mayor descendencia. Por otro lado, entre las cohortes de 1910 y 1925, la reducción de la infecundidad se produjo con fuerza en los niveles educativos más bajos (aquellas mujeres con educación primaria completa e incompleta), dinámica que no se observa en los otros niveles educativos en estas cohortes.

- $\quad$ Por último, se evidencia un aumento de los hijos sobrevivientes entre las cohortes de 1910 y 1930 , de mayor intensidad en los niveles educativos más bajos.

Estos determinantes demográficos se vieron influenciados por los siguientes factores contextuales:

- Las leyes sociales otorgaron protección social a un sector mayoritario de la población hasta entonces desprotegida, de esta forma contribuyeron a mejorar las malas las condiciones de vida reduciendo así los altos niveles de mortalidad.

- En el ámbito de la legislación laboral, políticas como la vivienda popular y el salario familiar, incentivaron el matrimonio como base de la familia industrial, que coincide con el aumento observado en la tasa bruta de nupcialidad.

- $\quad$ En este sentido, actuaron como un desincentivo para la participación de la mujer en el mercado de trabajo, y reforzaron la separación entre las esferas femeninas (domésticas y familiares) y masculinas (trabajo). En la mentalidad de la época, la industrialización requería de una población trabajadora que perseverara en el trabajo y tuviese necesidad de conservarlo, condiciones se cumplían con trabajadores comprometidos con una familia. De esta manera, observamos que la participación de la mujer en el mercado de trabajo fue menor en 1970 que a principios del siglo XX.

- En 1952 la creación del SNS trajo consigo la protección de la maternidad. Primero, durante los años cincuenta la preocupación se centró en el cuidado de la maternidad y del recién nacido, con el objetivo de reducir la mortalidad materna y neonatal. En segundo lugar, a mediados de la década de 1960 se procedió a la implementación de programas de planificación familiar con el objetivo de reducir la fecundidad. Aunque este objetivo no fue explicitado, su implementación fue efectiva en las cohortes que 
iniciaron la transición de fecundidad.

- $\quad$ La falta de cobertura del sistema educativo queda evidenciada en el censo de 1952 que mostraba niveles alarmantes de analfabetismo de la población en general y sobre todo en la población en edad escolar. No fue hasta la década de 1960 con el gobierno de Frei Montalva, que se invirtió fuertemente en la expansión de la cobertura del sistema educativo.

De esta manera, como hemos visto en el transcurso del artúculo, el esfuerzo realizado para modernizar la estructura económica y social del país, que en general fue exitoso en mejorar los indicadores de desarrollo, como los niveles de vida y bienestar social del conjunto de la población, no fue eficaz en modernizar el comportamiento reproductivo de las cohortes de 1910 a 1925. 


\section{REFERENCIAS}

Alba, F. (2014). Obras Escogidas de Victor L. Urquidi: Ensayos sobre Población y sociedad. México D.F.: El Colegio de México A.C.

Allende, S. (1939). La Realidad Médico-Social Chilena. México D.F.: Casa de Chile.

Anseoleaga, E. y Godoy, L. (2013). La maternidad y el trabajo en Chile: Discursos Actuales de Actores Sociales. Polis, 12 (35), 337-356.

Arellano, J. P. (1985). Políticas para Prometer el Ahorro en América Latina. Colección de Estudios CIEPLAN, 17, 127-151.

Bay, G., del Popolo, F. y Ferrando, D. (2003). Determinantes Próximos de La Fecundidad: Una Aplicación a Paises Latinoamericanos. Serie Población y Desarrollo, 43. Santiago de Chile: Centro Latinoamericano y Caribeño de Demografía (CELADE).

Binstock, G., y Cabella, W. (2011). La nupcialidad en el Cono Sur: Evolución reciente en la formación de uniones en Argentina, Chile y Uruguay. En G. Binstock, y V. J. Melo (Eds.), Nupcialidad y familia en la América Latina. Rio de Janeiro: Asociación Latinoamericana de Población.

Bongaarts, J. (1978). A Framework for Analyzing the Proximate Determinants of Fertility. Population and Development Review, 4 (1), 105-132.

Casas, L. y Herrera, T. (2013). Protección de la Maternidad Versus Derechos de Maternidad para las Trabajadoras en Chile: Una Reseña Histórica. Temas de Salud Reproductiva, 7, 58-67.

Chackiel, J. (2004). La Transición de Fecundidad en América Latina, 1950-2000. Papeles de Población, 41, 9-59.

Davis, B. K. (1963). Studies on the Termination of Pregnancy with Norethynodrel. Journal of Endocrinology, 27 (1), 99-106.

Davis, K., y Blake, J. (1956). Social Structure and Fertility: An Analytic Framework. Economic Development and Cultural Change, 4 (3), 211235. 
Devolder, D. y Merino, M. (2004). La Infecundidad y Fecundidad de las Familias desde una Perspectiva Longitudinal en los Paises Occidentales. Papers de Demografia, 250. Barcelona: Centre d'Estudis Demogràfics.

Dirección General de Estadísticas (1900-1951). Anuario Estadístico. Santiago de Chile: Dirección de Estadísticas y Censos, Ministerio de Economía.

- (1925). IX Censo de la Población de la República de Chile 1920. Santiago de Chile: Dirección de Estadísticas y Censos, Ministerio de Economía.

- (1931). X Censo de la Población de la República de Chile 1930. Santiago de Chile: Dirección de Estadísticas y Censos, Ministerio de Economía.

. (1941). XI Nacional de Población 1940. Dirección de Estadísticas y Censos, Ministerio de Economía.

. (1952). XII Censo General de Población y I de vivienda. Santiago de Chile: Servicio Nacional de Estadística y Censos. Ministerio de Economía.

. (1964). XIII Censo de Población y II de Vivienda (1960). Santiago de Chile: Dirección de Estadísticas y Censos, Ministerio de Economía.

Donoso, E. y Carvajal, J. (2009). Reducción de la Fecundidad y Envejecimiento de la Población de Mujeres Chilenas en Edad Fértil: 1990-2004. Revista Médica de Chile, 137 (6), 766-733.

Escobar, B. (2015). Female Entrepreneurship and Participation Rates in 19th Century Chile. Estudios de Economía, 42 (2), 67-91.

Ffrench-Davis, R. y Muñoz, O. (1990). Desarrollo Económico, Inestabilidad y Desequilibrios Políticos en Chile: 1950-1989. Colección de Estudios CIEPLAN, 28, 126-156.

Ffrench-Davis, R., Muñoz, O., Benavente, J. M. y Crespi, G. (2003). La Industrialización Chilena Durante el Proteccionismo (1940-1982). En E. Cárdenas, J. A. Ocampo, y R. Thorp (Eds.), Industrialización y Estado en la América Latina. La leyenda Negra de la Posguerra. México D.F.: Fondo de Cultura Económica 
Fernández, M. (2006). Los Usos de la Taberna: Renta Fiscal, Combate al Alcoholismo y Cacicazgo Político en Chile. 1870-1930. Historia, 39 (2), 369-429.

Fernández, M., Godoy, E., Herrera, P., Muñoz, J., Venegas, H. y Yáñez, J. (2008). Alcohol y Trabajo. El alcohol y la formación de las identidades laborales en Chile. Siglo XIX y XX. Osorno: Editorial Universidad de Los Lagos.

Godoy, L., Díaz, X. y Cardarelli, A. (2009). Imágenes sobre el Trabajo Femenino en Chile, 1880-2000. Universum, 24 (2), 74-93.

Illanes, M. A. (2004). Políticas Sociales y Modelos de Desarrollo: Puntos de Saturación Histórica. Chile, 1924-2003. Dimensión Histórica de Chile, 19, 149-205.

Instituto Nacional de Estadísticas (1952-1999). Anuario de Demografía. Santiago de Chile: Dirección de Estadísticas y Censos, Ministerio de Economía.

- (1970). XIV Censo Nacional de Población y III de Vivienda 1970. Santiago de Chile: Dirección de Estadísticas y Censos, Ministerio de Economía.

. (1982). XV Censo Nacional de Población y IV de Vivienda. Santiago de Chile: Dirección de Estadísticas y Censos, Ministerio de Economía.

. (1992). XVI Censo Nacional de Población y V de Vivienda 1992. Santiago de Chile: Ministerio de Economía.

Larrañaga, O. (2006a). Fertilidad en Chile 1960-2003, el Eslabón Perdido. Familia Modernización y Bienestar en Chile. Santiago de Chile: Taurus. . (2006b). Participación Laboral de la Mujer en Chile 19582003. Santiago de Chile: Departamento de Economía, Universidad de Chile.

Martínez Pizarro, J. (1998). La Transición Demográfica y las Diferencias Sociales de la Fecundidad y la Mortalidad Infantil en Chile. Santiago de Chile: Ministerio de Planificación. 
Molina, C. A. (2006). Antecedentes del Servicio Nacional de Salud. Historia de Debates y Contradicciones. Chile 1932-1952. Cuadernos Médico Sociales, 46 (4), 284-304.

Morgan, P. S. (1991). Late Nineteenth-And Early Twentieth-Century Childlessness. American Journal of Sociology, 97 (3), 779-807.

Nag, M., Abernethy, V., Bauwens, E., Browner, C., Lesthaeghe, R. y Mendieta, J. (1980). How Modernization Can Also Increase Fertility. Current Antropology, 21(5), 571-587.

Nicolau, R., Devolder, D. y Panareda, E. (2010b). La Modernización de los Comportamientos de Fecundidad en España Durante el siglo XX. Un Estudio a Nivel Provincial para las Generaciones en la Primera Mitad del Siglo XX. Papers Sociología, 95 (3), 633-653.

Ortiz, P., Uthoff, A., Gonzalez Cortéz, G., Correa, G., Errázuriz, M., Ramirez, V., y Tapia, R. (1978). Estrategia de desarrollo y transición demográfica el caso de Chile. Santiago de Chile: Centro Latinoamericano y Caribeño de Demográfia.

Olavarría, J. y Parrini, R. (2000). Masculinidadles. Identidad, Sexualidad y Familia. Santiago de Chile: FLACSO-Chile/Universidad Academia de Humanismo Cristiano.

Pardo, L. (1988). Una Revisión Histórica a la Participación de la Población en la Fuerza de Trabajo. Tendencias y Características de la Participación de la Mujer. Estudios de Economía, 15, 25-82.

Pérez-Brignoli, H. (2010). América Latina en la Transición Demográfica, 1800-1980. Población y Salud en Mesoamérica , 7 (2), 1-29.

Quilodrán, J. (2011). ¿Un Modelo de Nupcialidad Postransicional en América Latina? En G. Binstock, y. Melo Vieira, J. (Eds.), Nupcialidad y Familia en la América Latina Actual. Rio de Janeiro: Asociación Latinoamericana de Población.

Reher, D. y Requena, M. (2014). Was there a Mid-20th Century Fertility Boom in Latin America. Revista de Historia Económica. Journal of Iberian and Latin America Economic History, 32 (3), 319-350.

Rivero-Cantillano, R. (2016) El Cambio Demográfico en Chile y sus Efectos sobre la Fuerza de Trabajo (1934-2006). Tesis de Doctorado (Historia 
Económica), Universidad de Barcelona, España.

Rivero-Cantillano, R. y Spijker, J. (2015). Del Rejuvenecimiento al Envejecimiento de la Población ¿o Viceversa?: Chile en el Contexto de América Latina, 1950-2050. Notas de Población, N101, 127-155.

Rodriguez Vignoli, J. (2003). La Alta Fecundidad en América Latina y el Caribe: Un Riesgo en Transición. Santiago de Chile: Centro Latinoamericano y Caribeño de Demografía.

Rojas, C. (1994). Historia de la Política de Planificación Familiar en Chile: Un Caso Paradigmático. Debate Feminista, 10, 185-214.

Rojas, J. (2007). Los Derechos del Niño en Chile: Una Aproximación Histórica, 1910-1930. Historia, 40 (1), 129-164

Rosemblatt, K. (1995a). Masculinidad y Trabajo: El Salario Familiar y el Estado de Compromiso, 1930-1950. Revista Proposiciones, 26, 70-86.

. (1995b). Por un Hogar Bien Constituido. El Estado y su Política Familiar en los Frentes Populares. En L. Godoy, E. Hutchinson, K. Rosemblatt y Zárate, S. (Eds.), Disciplina y Desacato. Construcción de Identidad en Chile, Siglos XIX y XX. Santiago de Chile: SUR/CEDEM.

Rosero-Bixby, L. (1992). Las Tendencias de la Nupcialidad y la Transición de la Fecundidad en América Latina, Notas de Población, 55, 103-128

. (1996). Nuptiality Trends and Fertility Transition in Latin America. En Guzmán, J. M., Singh, S., Rodríguez, G. y Pantelides, E. (Eds.), The Fertility Transition in Latin America. Oxford: Oxford University Press.

Solsona, M. (1985). Fecundidad y Tamaño de la Familia en Chile 19601984. Santiago de Chile: Centro Latinoamericano y Caribeño de Demografía (CELADE).

Sunkel, O. (1969). Cambio y Frustración en Chile. En Veliz, C. (Ed.), Obstáculos para la Transformación de América Latina. México D.F.: Fondo de Cultura Económica.

Szot Meza, J. (2003). La Transición Demográfico-Epidemiológica en Chile 1960-2001. Revista Española de Salud Pública, 77 (5), 605-613. 
Valdés, X. (2007). Notas sobre la Metamorfosis de la Familia en Chile. Futuro de las Familias y Desafíos para las Politicas Públicas. Santiago de Chile: CEPAL/UNFPA.

Valdés, T. y Valdés, X. (2005). ¿Familia y Vida Privada: Transformaciones, Tensiones, Resistencias y Nuevos Sentidos? Santiago de Chile: FLACSO Chile.

Van de Kaa, D. J. (1997). Narraciones Ancladas: Historia y Resultados de Medio Siglo de Investigaciones sobre los Determinantes de la Fecundidad. Notas de Población, 66. Santiago de Chile: CEPAL.

Zabala de Cosío, M. (1992). La Transición Demográfica en América Latina y en Europa. Notas de Población, 20, 12-32.

Zárate, M. S. y Godoy, L. (2011). Madres y Niños en las Políticas del Servicio Nacional de Salud de Chile (1952-1964). História, Ciência, Saúde-Manguihos, 18, 131-151.

Zárate, S. (2007). Madres y Ciudad. La Red Urbana de la Asistencia Obstétrica Santiago 1900-1945. En. Valenzuela, J. (Ed), Historias Urbanas. Homenaje a Armando De Ramón (págs. 313-337). Santiago de Chile: Ediciones Universidad Católica de Chile.

Bases de datos

Integrated Public Use Microdata Series International (IPUMS-I). Microdatos Censales: https://www.ipums.org/

Centro de Microdatos de la Universidad de Chile, Encuesta de nEncuesta de Ocupación y Deosucpación del Gran Santiago http://www. microdatos.cl/Encuestas/Ocupacion/OcupacionDesocupacion?C=T2

Recibido: 11-04-2018

Aceptación de la versión final: 20-06-2018 